Pacific

Journal of

Mathematics

INNER QUASIDIAGONALITY AND STRONG NF ALGEBRAS

Bruce Blackadar and Eberhard KirchberG

Volume $198 \quad$ No. 2

April 2001 


\title{
INNER QUASIDIAGONALITY AND STRONG NF ALGEBRAS
}

\author{
Bruce Blackadar and Eberhard Kirchberg
}

\begin{abstract}
Continuing the study of generalized inductive limits of finitedimensional $C^{*}$-algebras, we define a refined notion of quasidiagonality for $C^{*}$-algebras, called inner quasidiagonality, and show that a separable $C^{*}$-algebra is a strong NF algebra if and only if it is nuclear and inner quasidiagonal. Many natural classes of NF algebras are strong NF, including all simple NF algebras, all residually finite-dimensional nuclear $C^{*}$-algebras, and all approximately subhomogeneous $C^{*}$-algebras. Examples are given of NF algebras which are not strong NF.
\end{abstract}

\section{Introduction.}

This paper is a sequel to Blackadar \& Kirchberg [BKb], to which we will frequently refer. In Blackadar \& Kirchberg, we studied a generalized inductive limit construction for $C^{*}$-algebras and gave various characterizations of $C^{*}$-algebras which can be written as generalized inductive limits of finitedimensional $C^{*}$-algebras. We recall the definitions for the convenience of the reader:

Definition 1.1. A separable $C^{*}$-algebra $A$ is an $M F$ algebra if it can be written as $\lim \left(A_{n}, \phi_{m, n}\right)$ for a generalized inductive system with the $A_{n}$ finite-dimensional. If the connecting maps $\phi_{m, n}$ can be chosen to be completely positive contractions, then $A$ is an $N F$ algebra, and $A$ is a strong $N F$ algebra if the $\phi_{m, n}$ can be chosen to be complete order embeddings.

There is a close relation between these notions and quasidiagonality and nuclearity: a (separable) $C^{*}$-algebra $A$ is an MF algebra if and only if it has an essential quasidiagonal extension by the compact operators $\mathbf{K}[\mathbf{B K} \mathbf{b}$, 3.2.2], and $A$ is an NF algebra if and only if it is nuclear and quasidiagonal $[\mathbf{B K b}, 5.2 .2]$. A number of other characterizations of $\mathrm{MF}, \mathrm{NF}$, and strong $\mathrm{NF}$ algebras are given in $[\mathbf{B K b}]$.

One major problem left unresolved in $[\mathbf{B K b}]$ is whether every NF algebra is a strong NF algebra. The purpose of this paper is to answer this question. We will characterize strong NF algebras in terms of a sharpened version of quasidiagonality we call inner quasidiagonality. The exact definition of inner quasidiagonality will be given in Section 2; roughly (and possibly not quite 
correctly), a $C^{*}$-algebra is inner quasidiagonal if it has a separating family of quasidiagonal irreducible representations.

As a consequence, we show that "most", but not all, NF algebras are strong NF. In particular, we show that the following $C^{*}$-algebras are strong NF:

All (separable) strongly quasidiagonal nuclear $C^{*}$-algebras.

All (separable) residually finite-dimensional nuclear $C^{*}$-algebras.

All (separable) approximately subhomogeneous $C^{*}$-algebras.

All prime antiminimal NF algebras.

All simple NF algebras.

We actually show that a prime strong NF algebra has a strong NF system $\left(A_{n}, \phi_{m, n}\right)$ in which each $A_{n}$ is a single matrix algebra.

On the other hand, there are NF algebras which are not strong NF: if $A$ is a (separable) prime nuclear $C^{*}$-algebra containing an ideal isomorphic to $\mathbf{K}$, then $A$ is strong $\mathrm{NF}$ if and only if its (unique) faithful irreducible representation is quasidiagonal. Thus the examples of $[\mathbf{B n}]$ and $[\mathbf{B n D}]$ are not strong NF. We also show that if $A$ is a separable nuclear $C^{*}$-algebra which is not strong NF, then $C A$ and $S A$ (which are NF by [BKb, 5.3.3]) are not strong $\mathrm{NF}$, answering $[\mathbf{B K b}, 6.2 .3(\mathrm{c})]$.

\section{Inner quasidiagonality.}

We begin by noting the following characterization of quasidiagonality from [Vo2, Theorem 1]:

Proposition 2.1. $A C^{*}$-algebra $A$ is quasidiagonal if and only if, for every $x_{1}, \ldots, x_{m} \in A$ and $\varepsilon>0$, there is a representation $\pi$ of $A$ on a Hilbert space $\mathbf{H}$ and a finite-rank projection $p \in \mathbf{B}(\mathbf{H})$ with $\left\|p \pi\left(x_{j}\right) p\right\|>\left\|x_{j}\right\|-\varepsilon$ and $\left\|\left[p, \pi\left(x_{j}\right)\right]\right\|<\varepsilon$ for all $j$.

Definition 2.2. A $C^{*}$-algebra $A$ is inner quasidiagonal if, for every $x_{1}, \ldots$, $x_{m} \in A$ and $\varepsilon>0$, there is a representation $\pi$ of $A$ on a Hilbert space $\mathbf{H}$ and a finite-rank projection $p \in \pi(A)^{\prime \prime} \subseteq \mathbf{B}(\mathbf{H})$ with $\left\|p \pi\left(x_{j}\right) p\right\|>\left\|x_{j}\right\|-\varepsilon$ and $\left\|\left[p, \pi\left(x_{j}\right)\right]\right\|<\varepsilon$ for all $j$.

It obviously suffices in this definition to assume that the $x_{j}$ have norm 1 .

The term "inner quasidiagonal" should really be "weakly inner quasidiagonal," but we have rejected this terminology on pedantic grounds.

An inner quasidiagonal $C^{*}$-algebra is obviously quasidiagonal. The converse is false (2.7).

Proposition 2.3. In the definition of inner quasidiagonality (2.2), the representation $\pi$ may be taken to be a direct sum of a finite number of mutually inequivalent irreducible representations. 
Proof. Suppose $A$ is inner quasidiagonal, and let $x_{1}, \ldots, x_{m} \in A$ and $\varepsilon>0$. Choose $\pi$ and $p$ as in 2.2. Let $z$ be the central support projection for $p$ in $\pi(A)^{\prime \prime}$; then $\pi(A)^{\prime \prime} z$ and hence also $\pi(A)^{\prime} z$ are type I von Neumann algebras with finite-dimensional centers. If $q$ is an abelian projection in $\pi(A)^{\prime}$ with central support $z$, then $\left.\pi\right|_{q \mathbf{H}}$ and $p q$ are the desired representation and projection.

This will be generalized later (3.7).

The following is an immediate consequence of the definition.

Proposition 2.4. If $A$ has a separating family of quasidiagonal irreducible representations, then $A$ is inner quasidiagonal. In particular, every residually finite-dimensional $C^{*}$-algebra is inner quasidiagonal.

We do not know whether the converse of 2.4 is true. (See note added in proof.) But an important special case of the converse is true, even in stronger form:

Proposition 2.5. If $A$ is separable and prime, then $A$ is inner quasidiagonal if and only if some (hence every) faithful irreducible representation of $A$ is quasidiagonal.

Proof. A $C^{*}$-algebra with a quasidiagonal faithful irreducible representation is obviously inner quasidiagonal. For the converse, consider the cases $A$ antiliminal (NGCR) and $A$ not antiliminal separately. If $A$ is antiliminal, separable, prime, and inner quasidiagonal, then $A$ is quasidiagonal, so by Voiculescu's Weyl-von Neumann Theorem [Vo1] every faithful representation not hitting the compacts (in particular, every faithful irreducible representation) of $A$ is quasidiagonal.

Now suppose $A$ is separable, prime, inner quasidiagonal, and not antiliminal. Then $A$ has an essential ideal isomorphic to $\mathbf{K}$, and has a unique faithful irreducible representation $\pi_{0}$ on a Hilbert space $\mathbf{H}_{0}$. Let $\left\{x_{i}\right\}$ be a dense sequence in $A$, and let $\left\{e_{i j}\right\}$ be a set of matrix units in $\mathbf{K} \subseteq A$. For each $n$ let $\pi_{n}$ and $p_{n}$ be as in 2.3 for the set $\left\{x_{1}, \ldots, x_{n}, e_{11}, \ldots, e_{n n}\right\}$ and for $\varepsilon=1 / n$. Then $\pi_{0}$ must be one of the irreducible subrepresentations of $\pi_{n}$ for each $n$ since $\left\|\pi_{n}\left(e_{11}\right)\right\|>\left\|e_{11}\right\|-1 / n \geq 0$. Let $q_{n}$ be the component of $p_{n}$ in $\pi_{0}$. Then, for any $j, \lim _{n \rightarrow \infty}\left\|q_{n} \pi_{0}\left(e_{j j}\right) q_{n}\right\|=1$ and $\lim _{n \rightarrow \infty}\left[q_{n}, \pi_{0}\left(x_{j}\right)\right]=0$, so $q_{n} \rightarrow 1_{\mathbf{H}_{0}}$ strongly and $\pi_{0}$ is quasidiagonal.

2.5 will be generalized in 3.18 .

Corollary 2.6. Every separable antiliminal quasidiagonal prime $C^{*}$-algebra is inner quasidiagonal. Every separable simple quasidiagonal $C^{*}$-algebra is inner quasidiagonal.

Example 2.7. (a) The examples of $[\mathbf{B n}]$ and $[\mathbf{B n D}]$ are quasidiagonal, but not inner quasidiagonal by 2.5 . 
We recall for the reader that the example of $[\mathbf{B n}]$ is an essential extension of the continuous functions on the real projective plane $\mathbf{R} P^{2}$ by the compacts:

$$
0 \rightarrow \mathbf{K} \rightarrow A \rightarrow C\left(\mathbf{R} P^{2}\right) \rightarrow 0 .
$$

The examples of $[\mathbf{B n D}]$ are slight variations of this, and have the additional feature that the extension has real rank zero.

(b) A similar example is the $C^{*}$-algebra generated by the direct sum of the unilateral shift $s$ and its adjoint. The $C^{*}$-algebra is not prime; we have the extension

$$
0 \rightarrow \mathbf{K} \oplus \mathbf{K} \rightarrow C^{*}\left(s \oplus s^{*}\right) \rightarrow C(\mathbf{T}) \rightarrow 0 .
$$

$C^{*}(s \oplus s)$ is quasidiagonal, but has only two irreducible representations nonzero on the $\mathbf{K} \oplus \mathbf{K}$, neither of which is quasidiagonal; hence it is easily seen not to be inner quasidiagonal.

Proposition 2.8. Let $A$ be a separable $C^{*}$-algebra. The following are equivalent:

(i) Every quotient of $A$ is inner quasidiagonal.

(ii) Every primitive quotient of $A$ is inner quasidiagonal.

(iii) Every irreducible representation of $A$ is quasidiagonal.

(iv) $A$ is strongly quasidiagonal, i.e., every representation of $A$ is quasidiagonal.

Proof. (iv) $\Rightarrow$ (iii) $\Rightarrow$ (i) $\Rightarrow$ (ii) is trivial, and (ii) $\Rightarrow$ (iii) is 2.5. To show (iii) $\Rightarrow$ (iv), let $\pi$ be a representation, which we may assume is nondegenerate, of $A$ on a Hilbert space $\mathbf{H}$, which we may assume is separable. Let $J=\pi^{-1}(\mathbf{K})$. Then $\pi=\pi_{1} \oplus \pi_{2}$, where $\left.\pi_{1}\right|_{J}$ is nondegenerate and $\pi_{2}(J)=0$. Then $\pi_{1}$ is a direct sum of irreducible representations, hence quasidiagonal, and $\pi_{2}$ is quasidiagonal by Voiculescu's Weyl-von Neumann Theorem since $\pi_{2}(A)$ is a quasidiagonal $C^{*}$-algebra by (i).

Proposition 2.9. Let $A$ be a $C^{*}$-algebra, and $J_{1}, J_{2}$ ideals of $A$. Set $J=$ $J_{1} \cap J_{2}$. If $A / J_{1}$ and $A / J_{2}$ are inner quasidiagonal, then $A / J$ is inner quasidiagonal.

Proof. We may clearly assume $J=0$. Let $x_{1}, \ldots, x_{m} \in A$ and $\varepsilon>0$. Let $\rho_{k}(k=1,2)$ be the quotient map from $A$ to $A / J_{k}$. Then, for each $j,\left\|x_{j}\right\|=$ $\max \left(\left\|\rho_{1}\left(x_{j}\right)\right\|,\left\|\rho_{2}\left(x_{j}\right)\right\|\right)$. By reordering we may assume $\left\|\rho_{1}\left(x_{j}\right)\right\|=\left\|x_{j}\right\|$ for $1 \leq j \leq r$ and $\left\|\rho_{2}\left(x_{j}\right)\right\|=\left\|x_{j}\right\|>\left\|\rho_{1}\left(x_{j}\right)\right\|$ for $r+1 \leq j \leq m$. We may also assume $\varepsilon$ is small enough that $\left\|\rho_{1}\left(x_{j}\right)\right\|<\left\|x_{j}\right\|-\varepsilon$ for $r+1 \leq j \leq m$.

Let $\sigma_{k}(k=1,2)$ be representations of $A / J_{k}$ as in 2.3 for $\left\{\rho_{k}\left(x_{1}\right), \ldots\right.$, $\left.\rho_{k}\left(x_{m}\right)\right\}$ and the given $\varepsilon$, with projections $p_{k}$. Let $\sigma_{0}$ be the subrepresentation of $\sigma_{2}$ consisting of those irreducible subrepresentations $\sigma$ such that $\left\|p \sigma\left(\rho_{2}\left(x_{j}\right)\right) p\right\|>\left\|x_{j}\right\|-\varepsilon$ for at least one $j, r+1 \leq j \leq m$ (where $p$ denotes the $\sigma$-component of $\left.p_{2}\right)$, and $p_{0}$ the component of $p_{2}$ in $\sigma_{0}$. Then all of the 
irreducible subrepresentations of $\sigma_{0}$, regarded as representations of $A$, are disjoint from $\sigma_{1} \circ \rho_{1}$ since each such $\sigma$ satisfies $\left\|\sigma\left(\rho_{2}\left(x_{j}\right)\right)\right\|>\left\|\rho_{1}\left(x_{j}\right)\right\|$ for at least one $j$. Thus $\pi=\sigma_{1} \circ \rho_{1} \oplus \sigma_{0} \circ \rho_{2}$ and $p=p_{1} \oplus p_{0}$ have the desired properties from 2.2 .

Corollary 2.10. $A C^{*}$-algebra $A$ is inner quasidiagonal if $A$ contains a collection $\left\{J_{i}\right\}$ of ideals with $A / J_{i}$ inner quasidiagonal for all $i$ and $\cap J_{i}=0$.

Proof. A direct proof can be given along the lines of the proof of 2.9. Alternatively, note that the result is immediate from the definition of inner quasidiagonality if the $J_{i}$ are directed by inclusion. In general, let $J_{i_{1}, \ldots, i_{n}}=J_{i_{1}} \cap \ldots \cap J_{i_{n}}$, and use 2.9 to conclude that $A / J_{i_{1}, \ldots, i_{n}}$ is inner quasidiagonal.

Remark 2.11. It is obvious from the definition that if $\left\{A_{i}, \phi_{i j}\right\}$ is an (ordinary) inductive system (indexed by any directed set) of inner quasidiagonal $C^{*}$-algebras with injective connecting maps $\phi_{i j}$, then the inductive limit is inner quasidiagonal. It is not true that the inductive limit of an inductive system with noninjective connecting maps is necessarily inner quasidiagonal, as the the following example shows. (It is an inductive system with surjective connecting maps.) The same question can be asked about quasidiagonality, where it appears to have a positive answer; the closely related classes of MF and NF algebras are closed under (ordinary) inductive limits with noninjective connecting maps, as well as certain generalized inductive limits [BKb, 3.4.4 and 5.3.5].

Example 2.12. Let $B$ be a (separable) quasidiagonal $C^{*}$-algebra which is not inner quasidiagonal, e.g., the example of $[\mathbf{B n}](2.7)$. Let $\pi$ be a faithful representation of $B$ of infinite multiplicity on a separable Hilbert space $\mathbf{H}_{0}$; then $\pi$ is quasidiagonal. Let $\left\langle\mathbf{H}_{n}\right\rangle_{n \geq 1}$ be a sequence of separable infinite-dimensional Hilbert spaces, with distinguished unit vectors $\xi_{n}$, and set $\mathbf{H}^{(n)}=\otimes_{k \geq n}\left(\mathbf{H}_{k}, \xi_{k}\right)$. If $\mathbf{H}=\mathbf{H}_{0} \otimes \mathbf{H}^{(1)}$, define $C^{*}$-subalgebras of $\mathbf{B}(\mathbf{H})$ by $C_{n}=\mathbf{K}\left(\mathbf{H}_{0}\right) \otimes 1_{\mathbf{H}_{1}} \otimes \cdots \otimes 1_{\mathbf{H}_{n-1}} \otimes \mathbf{K}\left(\mathbf{H}^{(n)}\right)\left[C_{1}=\mathbf{K}(\mathbf{H})\right], J_{n}=$ $C_{1}+\cdots+C_{n}, A_{n}=J_{n}+\rho(B), J=\left[\cup J_{n}\right]^{-}, A=J+\rho(B)=\left[\cup A_{n}\right]^{-}$, where $\rho=\pi \otimes 1_{\mathbf{H}^{(1)}}$. Then $\left\langle J_{n}\right\rangle$ is an increasing sequence of ideals of $A$. Each $A_{n}$ is inner quasidiagonal by repeated applications of 2.5 ; thus $A$ is inner quasidiagonal by 2.11. $A / J_{n}$ is isomorphic to $A$ for any $n$, hence inner quasidiagonal; but $A / J \cong B$ is not inner quasidiagonal.

\section{Variations and technicalities.}

A somewhat cleaner alternative definition of inner quasidiagonality can be given using the socle of the bidual. See $[\mathbf{B o D}]$ for the general theory of socles of Banach algebras.

Definition 3.1. If $B$ is a $C^{*}$-algebra, then a projection $p \in B$ is in the socle of $B$ if $p B p$ is finite-dimensional. 
Proposition 3.2. $A C^{*}$-algebra $A$ is inner quasidiagonal if and only if, for any $x_{1}, \ldots, x_{m} \in A$ and $\varepsilon>0$, there is a projection $p$ in the socle of $A^{* *}$ with $\left\|p x_{j} p\right\|>\left\|x_{j}\right\|-\varepsilon$ and $\left\|\left[p, x_{j}\right]\right\|<\varepsilon$ for all $j$.

Proposition 3.3. Let $B$ be $a C^{*}$-algebra, $x \in B$, and $p$ a projection in $B$. Then

$$
\begin{aligned}
\|[x, p]\| & =\max (\|(1-p) x p\|,\|p x(1-p)\|) \\
& =\max \left(\left\|p x^{*} x p-p x^{*} p x p\right\|^{1 / 2},\left\|p x x^{*} p-p x p x^{*} p\right\|^{1 / 2}\right) .
\end{aligned}
$$

Proof. Set $a=(1-p) x p, b=p x(1-p)$. Then calculation shows that $[x, p]^{*}[x, p]=a^{*} a+b^{*} b$, so $\|[x, p]\|^{2}=\left\|a^{*} a+b^{*} b\right\|=\max \left(\left\|a^{*} a\right\|,\left\|b^{*} b\right\|\right)$ since $a^{*} a$ and $b^{*} b$ are orthogonal. Also, $\|[x, p]\|^{2}=\max \left(\left\|a^{*} a\right\|,\left\|b b^{*}\right\|\right)$, and $a^{*} a=p x^{*} x p-p x^{*} p x p$ and $b b^{*}=p x x^{*} p-p x p x^{*} p$.

The following fact, which is a slight sharpening of the Kadison Transitivity Theorem, follows immediately from [Pd, 2.7.5 and 3.11.9].

Proposition 3.4. Let $A$ be a $C^{*}$-algebra, and $p$ a projection in the socle of $A^{* *}$. Set $N_{p}=\{x \in A:[p, x]=0\}=\{p\}^{\prime} \cap A$. Then

(a) $p N_{p}=p N_{p} p=p A^{* *} p(=p A p)$.

(b) The weak closure of $N_{p}$ in $A^{* *}$ is $p A^{* *} p+(1-p) A^{* *}(1-p)$.

Corollary 3.5. Let $A, p, N_{p}$ be as in 3.4, and let $x \in A$. Then $d\left(x, N_{p}\right)=$ $\|[x, p]\|$.

Proof. $d\left(x, N_{p}\right)=d\left(x, N_{p}^{* *}\right)$ by the Hahn-Banach Theorem. We have $y=$ $\operatorname{pxp}+(1-p) x(1-p) \in N_{p}^{* *}$ by 3.4 , and

$$
\begin{aligned}
\|x-y\| & =\|(1-p) x p+p x(1-p)\| \\
& =\max (\|(1-p) x p\|,\|p x(1-p)\|)=\|[x, p]\| .
\end{aligned}
$$

So $d\left(x, N_{p}\right) \leq\|[x, p]\|$. Conversely, if $y \in N_{p}$, then

$$
\begin{aligned}
\|[x, p]\| & =\|[x-y, p]\| \\
& =\max (\|(1-p)(x-y) p\|,\|p(x-y)(1-p)\|) \leq\|x-y\| .
\end{aligned}
$$

We now show that in many instances, the study of inner quasidiagonality can be reduced to the separable case.

Proposition 3.6. Let $A$ be an inner quasidiagonal $C^{*}$-algebra, and $B$ a separable $C^{*}$-subalgebra of $A$. Then there is a separable inner quasidiagonal $C^{*}$-subalgebra $E$ of $A$ containing $B$.

Proof. We show that if $x_{1}, \ldots, x_{m} \in B$ and $\varepsilon>0$, then there is a separable $C^{*}$-subalgebra $D$ of $A$ containing $B$, and a projection $q$ in the socle of $D^{* *}$ with $\left\|\left[q, x_{j}\right]\right\|<\varepsilon$ and $\left\|q x_{j} q\right\|>\left\|x_{j}\right\|-\varepsilon$ for $1 \leq j \leq m$. Choose a projection 
$p$ in the socle of $A^{* *}$ with $\left\|\left[p, x_{j}\right]\right\|<\varepsilon / 2$ and $\left\|p x_{j} p\right\|>\left\|x_{j}\right\|-\varepsilon / 2$ for all $j$. Let $C$ be a separable $C^{*}$-subalgebra of $N_{p}$ with $d\left(x_{j}, C\right)=d\left(x_{j}, N_{p}\right)$ for all $j$ and $p C=p N_{p}=p A p$, and let $D$ be the $C^{*}$-subalgebra of $A$ generated by $B$ and $C$. Then $D$ is separable. Regard the homomorphism $\pi: C \rightarrow p C=p A p$ as a representation of $C$ on a finite-dimensional Hilbert space $\mathbf{H}$, and extend $\pi$ to a representation $p$ of $D$ on a larger Hilbert space $\widetilde{\mathbf{H}}$ such that $\rho$ is a direct sum of finitely many mutually inequivalent irreducible representations (by extending each irreducible subrepresentation of $\pi$ to an irreducible representation of $D$ and identifying together equivalent ones if necessary). If $Q$ is the projection from $\widetilde{\mathbf{H}}$ onto $\mathbf{H}$, then $Q$ may be regarded as a projection $q$ in the socle of $D^{* *}$, and $C \subseteq N_{q}$. We have by 3.5, for each $j$,

$$
\left\|\left[q, x_{j}\right]\right\|=d\left(x_{j}, N_{q}\right) \leq d\left(x_{j}, C\right)=\left\|\left[p, x_{j}\right]\right\|<\varepsilon .
$$

Also, there is an isometry $\psi$ from $q D q$ to $p A p$ induced by $\rho$, and $\psi(q a q)=$ pap for $a \in C$. Since $d\left(x_{j}, C\right)<\varepsilon / 2$, we have, for each $j$,

$$
\left\|q x_{j} q\right\|>\left\|p x_{j} p\right\|-\varepsilon / 2>\left\|x_{j}\right\|-\varepsilon .
$$

As a consequence, if $B=B_{1}$ is a separable $C^{*}$-subalgebra of $A$, then there is a larger separable $C^{*}$-subalgebra $B_{2}$ of $A$ such that, if $x_{1}, \ldots, x_{m} \in B_{1}$ and $\varepsilon>0$, there is a projection $q$ in the socle of $B_{2}^{* *}$ with $\left\|\left[q, x_{j}\right]\right\|<\varepsilon$ and $\left\|q x_{j} q\right\|>\left\|x_{j}\right\|-\varepsilon$ for $1 \leq j \leq m$. Iterating this construction, obtain an increasing sequence $\left\langle B_{n}\right\rangle$, and set $E=\left[\cup B_{n}\right]^{-}$. Then $E$ is separable and inner quasidiagonal.

Separability is nice because of the following characterizations of inner quasidiagonality. By a slight extension of usual terminology, we will say an irreducible representation $\pi$ of a $C^{*}$-algebra $A$ is a $G C R$ representation if $\pi(A)$ contains the compact operators.

Proposition 3.7. Let $A$ be a separable $C^{*}$-algebra. The following are equivalent:

(i) A is inner quasidiagonal.

(ii) Given $x_{1}, \ldots, x_{m} \in A$ and $\varepsilon>0$, there is an irreducible representation $\pi$ of $A$ on a Hilbert space $\mathbf{H}$ and a finite-rank projection $p \in \mathbf{B}(\mathbf{H})$ such that $\left\|\left[p, \pi\left(x_{j}\right)\right]\right\|<\varepsilon$ for $1 \leq j \leq m$ and $\left\|p \pi\left(x_{1}\right) p\right\|>\left\|x_{1}\right\|-\varepsilon$.

(iii) There is a sequence of irreducible representations $\left\langle\pi_{n}\right\rangle$ of $A$ on Hilbert spaces $\mathbf{H}_{n}$, and finite-rank projections $p_{n} \in \mathbf{B}\left(\mathbf{H}_{n}\right)$, such that $\left\|\left[p_{n}, \pi_{n}(x)\right]\right\| \rightarrow 0$ and $\lim \sup \left\|p_{n} \pi_{n}(x) p_{n}\right\|=\|x\|$ for all $x \in A$.

(iv) There is a sequence of irreducible representations $\left\langle\pi_{n}\right\rangle$ of $A$ on Hilbert spaces $\mathbf{H}_{n}$, and finite-rank projections $p_{n} \in \mathbf{B}\left(\mathbf{H}_{n}\right)$, such that $\left\|\left[p_{n}, \pi_{n}(x)\right]\right\| \rightarrow 0$ and $\lim \sup \left\|p_{n} \pi_{n}(x) p_{n}\right\|=\|x\|$ for all $x \in A$, such that any representation occuring more than once (up to equivalence) in the sequence is quasidiagonal and GCR. 
Proof. (i) $\Rightarrow$ (ii) $\Leftrightarrow$ (iii) are obvious. To prove (iv) $\Rightarrow$ (i), let $x_{1}, \ldots, x_{m} \in A$ and $\varepsilon>0$, and let $\left\langle\left(\pi_{n}, p_{n}\right)\right\rangle$ be as in (iv). Choose $\left(\pi_{n_{1}}, p_{n_{1}}\right), \ldots,\left(\pi_{n_{m}}, p_{n_{m}}\right)$ with $\left\|p_{n_{k}} \pi_{n_{k}}\left(x_{k}\right) p_{n_{k}}\right\|>\left\|x_{k}\right\|-\varepsilon$ and $\left\|\left[\pi_{n_{k}}\left(x_{j}\right), p_{n_{k}}\right]\right\|<\varepsilon$ for all $j$ and $k$. If there is a subset $F$ of $\{1, \ldots, m\}$ with $|F|>1$ such that $\pi_{n_{k}}=\pi_{0}$ for $n \in F$, then $\pi_{0}$ is quasidiagonal, so there is a finite-rank projection $q$ in $\pi_{0}(A)^{\prime \prime}$ with $\left\|q \pi_{0}\left(x_{k}\right) q\right\|>\left\|x_{k}\right\|-\varepsilon$ for all $k \in F$. Replace $\oplus_{k=1}^{m}\left(\pi_{n_{k}}, p_{n_{k}}\right)$ by $\left(\pi_{0}, q\right) \oplus \oplus_{k \in F}\left(\pi_{n_{k}}, p_{n_{k}}\right)$. Repeating the process finitely many times if necessary, we obtain a representation as in 2.3.

So we need only prove (ii) $\Rightarrow$ (iv). Let $\left\langle x_{k}\right\rangle$ be a dense sequence in $A$, and choose a doubly indexed sequence $\left\langle\rho_{i j}\right\rangle(1 \leq k \leq j)$ of irreducible representations of $A$ on $\mathbf{H}_{j k}$, and finite-rank projections $q_{j k} \in \mathbf{B}\left(\mathbf{H}_{j k}\right)$, such that $\left\|\left[q_{j k}, \rho_{j k}\left(x_{i}\right)\right]\right\|<j^{-1}$ for $1 \leq i \leq j$ and $\left\|q_{j k} \rho_{j k}\left(x_{i}\right) q_{j k}\right\|>\left\|x_{k}\right\|-j^{-1}$. (Note that this sequence satisfies the conditions of (iii).) The rest of the proof will consist of two parts.

(1) We first show that if infinitely many $\rho_{j k}$ are equivalent to a single $\rho$, then either $\rho$ is quasidiagonal and GCR or the sequence can be modified to a new sequence in which no $\rho_{j k}$ is equivalent to $\rho$. If $\rho(A) \cap \mathbf{K}=\{0\}$, then there are infinitely (in fact, uncountably) many mutually inequivalent irreducible representations of $A$ with the same kernel as $\rho$ (see Appendix), and if $\pi$ is any such representation, on a Hilbert space $\widetilde{\mathbf{H}}$, and $\varepsilon>0$, then for any $j, k$ with $\rho_{j k} \approx \rho$, by [Vo1, Lemma 1] there is a unitary $u$ from $\mathbf{H}_{j k}$ to $\widetilde{\mathbf{H}}$ and a finite-rank projection $p \in \mathbf{B}(\widetilde{\mathbf{H}})$ such that

$$
\left\|u q_{j k} \rho_{j k}\left(x_{i}\right) q_{j k} u^{*}-p \pi\left(x_{i}\right) p\right\|<\varepsilon
$$

for $1 \leq i \leq j$. For sufficiently small $\varepsilon,\left(\rho_{j k}, q_{j k}\right)$ can be replaced by $(\pi, p)$, and a different $\pi$ can be used for each $\rho_{j k}$ equivalent to $\rho$.

Now suppose that $\rho$ is GCR. If $J=\operatorname{ker} \rho$, then there is an ideal $K$ of $A$ with $K / J$ essential in $A / J$ and isomorphic to $\mathbf{K}$. By identifying $\mathbf{H}_{j k}$ with $\mathbf{H}$ (the Hilbert space on which $\rho$ acts) for each $\rho_{j k}$ equivalent to $\rho$, the projections $q_{j k}$ become a sequence $\left\langle r_{n}\right\rangle$ of finite-rank projections in $\mathbf{B}(\mathbf{H})$ which asymptotically commute in norm with $\rho(A)$, and in particular with $\mathbf{K}$; thus the only possible weak operator limit points of the sequence are 0 and 1 [any limit point must be a scalar by irreducibility, and if $\lambda 1$ is a limit point and $p$ a finite-rank projection, then $\left\langle p r_{n} p\right\rangle$ has a subsequence converging in norm to $\lambda p$; but $p r_{n} p$ is approximately a projection for large $n$, so any norm limit point must be a projection]. If 1 is a limit point, then there is a subsequence of $\left\langle r_{n}\right\rangle$ converging weakly, hence strongly, to 1 , so $\rho$ is quasidiagonal.

If 1 is not a limit point, then $r_{n} \rightarrow 0$ weakly, hence strongly, and so $r_{n} a r_{n} \rightarrow 0$ in norm for all $a \in \mathbf{K}$, i.e., $\left\|r_{n} \rho(x) r_{n}\right\| \rightarrow 0$ for all $x \in K$. Fix $x_{0} \in K$ of norm 1 . We can then find a subsequence $\left\langle\rho_{n}\right\rangle$ of $\left\{\rho_{j k}\right\}$, with associated Hilbert spaces $\mathbf{H}_{n}$ and projections $q_{n}$, such that $\left\|\left[q_{n}, \rho_{n}(x)\right]\right\| \rightarrow 0$ for all $x \in A,\left\|q_{n} \rho_{n}\left(x_{0}\right) q_{n}\right\| \rightarrow 1$, and such that $\rho_{n}$ is not equivalent to $\rho$ 
for any $n$. Define $\phi: A \rightarrow \prod q_{n} \mathbf{B}\left(\mathbf{H}_{n}\right) q_{n}$ by $\phi(x)=\prod q_{n} \rho_{n}(x) q_{n} . \phi$ then drops to a ${ }^{*}$-homomorphism $\sigma$ from $A$ to $\left(\prod q_{n} \mathbf{B}\left(\mathbf{H}_{n}\right) q_{n}\right) /\left(\oplus q_{n} \mathbf{B}\left(\mathbf{H}_{n}\right) q_{n}\right)$. Set $I=\operatorname{ker} \sigma$. Then $I \cap K \subseteq J$ since $x_{0} \notin I$, so $(I+J) / J$ is an ideal of $A / J$ orthogonal to $K / J$. But $K / J$ is essential in $A / J$, so $(I+J) / J=0$, $I \subseteq J$, and so $\|\sigma(x)\| \geq\|\rho(x)\|$ for all $x \in A$. Thus the subsequence of $\left\{\rho_{j k}\right\}$ consisting of those which are not equivalent to $\rho$ still satisfies the conditions of (iii), and a smaller subsequence will have the same specific properties as the full double sequence $\left\{\rho_{j k}\right\}$ if suitably reindexed.

(2) We now construct a doubly indexed sequence $\left\langle\left(\pi_{j k}, p_{j k}\right)\right\rangle$ satisfying the conditions of (iv). Suppose $\left\{\left(\pi_{j k}, p_{j k}\right)\right\}$ have been chosen from among the $\left(\rho_{n r}, q_{n r}\right)$ for $1 \leq k \leq j<m$, satisfying the following properties:

(a) $\left\|\left[p_{j k}, \pi_{j k}\left(x_{i}\right)\right]\right\|<j^{-1}$ for $1 \leq i \leq j$.

(b) $\left\|p_{j k} \pi_{j k}\left(x_{i}\right) p_{j k}\right\|>\left\|x_{k}\right\|-j^{-1}$.

(c) No irreducible representation occurs more than once among the $\pi_{j k}$ chosen so far unless it is quasidiagonal and GCR.

If $m=1$, choose $\left(\pi_{11}, p_{11}\right)$ to satisfy (a) and (b). If $m>1$, the $\pi_{j k}$ already chosen come from the $\rho_{n r}$ for $n \leq n_{0}$ for some $n_{0}$. The tail $\left\{\rho_{n r}: n>n_{0}\right\}$ can be modified as in (1), and further truncated by increasing $n_{0}$ if necessary, so that none of the $\left\{\pi_{j k}: 1 \leq k \leq j<m\right\}$ occurs in the tail unless it is quasidiagonal and GCR. Then a suitable element of the modified tail satisfies (a) and (b) and can be chosen as $\pi_{m 1}$. After again modifying and truncating the tail to eliminate subsequent appearances of $\pi_{m 1}$ if necessary, $\pi_{m 2}$ can be chosen. The process can be continued inductively to get the desired representations and projections.

This completes the proof.

Lemma 3.8. Let $A$ be a $C^{*}$-algebra. Then, for any $k, A$ is inner quasidiagonal if and only if $M_{k}(A)=A \otimes \mathbf{M}_{k}$ is inner quasidiagonal. The projections for $A \otimes \mathbf{M}_{k}$ may be chosen of the form $p \otimes 1_{k}$, where $p$ is in the socle of $A^{* *}$.

Proof. Suppose $A$ is inner quasidiagonal. It follows from 3.6 that we may assume $A$ is separable (or the following argument may be easily modified to apply to the nonseparable case). Let $\left\langle\left(\pi_{n}, p_{n}\right)\right\rangle$ be a sequence as in 3.7 (iii). The map $\phi: A \rightarrow \prod p_{n} \mathbf{B}\left(\mathbf{H}_{n}\right) p_{n}$ given by $\phi(x)=\prod p_{n} \pi_{n}(x) p_{n}$ drops to an injective *-homomorphism $\sigma$ from $A$ to $\left(\prod p_{n} \mathbf{B}\left(\mathbf{H}_{n}\right) p_{n}\right) / \oplus p_{n} \mathbf{B}\left(\mathbf{H}_{n}\right) p_{n}$, and hence $\sigma \otimes 1_{k}$ is an injective *-homomorphism from $A \otimes M_{k}$ to

$$
\begin{aligned}
& {\left[\left(\prod p_{n} \mathbf{B}\left(\mathbf{H}_{n}\right) p_{n}\right) / \oplus p_{n} \mathbf{B}\left(\mathbf{H}_{n}\right) p_{n}\right] \otimes M_{k}} \\
& \cong\left(\prod\left(p_{n} \otimes 1_{k}\right)\left[\mathbf{B}\left(\mathbf{H}_{n}\right) \otimes M_{k}\right]\left(p_{n} \otimes 1_{k}\right)\right) / \oplus\left(p_{n} \otimes 1_{k}\right) \\
& \quad \cdot\left[\mathbf{B}\left(\mathbf{H}_{n}\right) \otimes M_{k}\right]\left(p_{n} \otimes 1_{k}\right) .
\end{aligned}
$$

The converse is trivial. 
Proposition 3.9. Let $A$ and $B$ be $C^{*}$-algebras, with $A$ inner quasidiagonal and $B$ residually finite-dimensional (e.g., commutative). Then $A \otimes B=$ $A \otimes_{\min } B$ is inner quasidiagonal.

Proof. Let $\{J\}$ be a collection of ideals of $B$ with intersection 0 , with $B / J_{i}$ finite-dimensional for all $i$. Then $\left\{A \otimes J_{i}\right\}$ is a collection of ideals of $A \otimes B$, with intersection 0 , and $(A \otimes B) /\left(A \otimes J_{i}\right)=A \otimes\left(B / J_{i}\right)$ is a finite direct sum of matrix algebras over $A$, hence inner quasidiagonal.

We will prove a partial converse to 3.9 in 3.10 below. We believe that a tensor product of any two inner quasidiagonal $C^{*}$-algebras is inner quasidiagonal, at least if one of the factors is nuclear. This would follow if the converse to 2.4 is true. (See note added in proof.) Note that a tensor product of strong NF algebras is strong NF (5.17).

Proposition 3.10. Let $A$ and $C$ be $C^{*}$-algebras, with $C$ commutative. If $A \otimes C$ is inner quasidiagonal, then $A$ is also inner quasidiagonal.

Proof. We reduce to the case where $A$ is separable. If $A \otimes C$ is inner quasidiagonal, and $S$ is a countable subset of $A$, then by 3.6 and an obvious additional construction we may construct an increasing sequence $\left\langle B_{n}\right\rangle$ of separable $C^{*}$-subalgebras of $A \otimes C$ such that $B_{n}$ is inner quasidiagonal for $n$ odd and $B_{n}=D_{n} \otimes E_{n}$ for $n$ even, for separable $C^{*}$-subalgebras $D_{n}$ of $A$ containing $S$ and $E_{n}$ of $C$. Then $B=\left[\cup B_{n}\right]^{-}$is separable, inner quasidiagonal, and equal to $D \otimes E$ for $D=\left[\cup D_{n}\right]^{-} \subseteq A$, which contains $S$, and $E=\left[\cup E_{n}\right]^{-} \subseteq C$.

Now suppose $A$ is separable. Write $C=C_{0}(X)$ for a locally compact Hausdorff space $X$; then $A \otimes C=C_{0}(X, A)$. Suppose $A \otimes C$ is inner quasidiagonal; let $x_{1}, \ldots, x_{m} \in A$, all of norm 1 , and $0<\varepsilon<1$. Choose $\delta>0$ such that $\frac{\delta}{1-\delta}<\varepsilon$. Let $U$ be an open set in $X$ with compact closure, $g \in C_{0}(X)$ of norm 1 supported in $U$, and $f \in C_{0}(X)$ of norm 1 and identically 1 on $U$, with $f$ and $g$ taking values in $[0,1]$. Let $V=\{x: g(x)>1-\delta\} \subseteq U$. Let $\pi$ be an irreducible representation of $A \otimes C=C_{0}(X, A)$ and $p$ a finiterank projection such that $\left\|p \pi\left(x_{1} \otimes g\right) p\right\|>1-\delta$ and $\left[p, \pi\left(x_{j} \otimes g\right)\right]<\delta$ for all $j$. Then $\pi$ is supported on a point $x_{0}$ of $V$, so $\pi$ may be regarded as a representation $\rho$ of $A$ by $\rho(x)=\pi(x \otimes f)$. For each $j, \pi\left(x_{j} \otimes f\right)$ is a scalar multiple of $\pi\left(x_{j} \otimes g\right)$, with a scalar $\lambda=g\left(x_{0}\right)^{-1}$ satisfying $1 \leq \lambda \leq(1-\delta)^{-1}$; thus $\left\|\left[q, \pi\left(x_{j} \otimes f\right)\right]\right\| \leq(1-\delta)^{-1}\left\|\left[q, \pi\left(x_{j} \otimes g\right)\right]\right\|<\varepsilon$, and $\left\|p \pi\left(x_{1} \otimes f\right) p\right\| \geq\left\|p \pi\left(x_{j} \otimes g\right) p\right\|>1-\delta>1-\varepsilon$. Thus $\rho$ and $p$ satisfy condition (ii) of 3.7 , so $A$ is inner quasidiagonal.

Corollary 3.11. $S A$ is inner quasidiagonal if and only if $A$ is inner quasidiagonal, and similarly for $C A$.

Proof. Combine 3.10 with 3.9 . 
Remark 3.12. 3.11 shows that unlike quasidiagonality [Vo2], inner quasidiagonality is not a homotopy invariant for $C^{*}$-algebras.

We have the following refinement of the notion of inner quasidiagonality:

Definition 3.13. Let $A$ be a $C^{*}$-algebra, and $z$ a central projection in $A^{* *}$. Then $A$ is $z$-inner quasidiagonal if, for any $x_{1}, \ldots, x_{m} \in A$ and $\varepsilon>0$, there is a projection $p$ in the socle of $z A^{* *}$ with $\left\|p x_{j} p\right\|>\left\|x_{j}\right\|-\varepsilon$ and $\left\|\left[p, x_{j}\right]\right\|<\varepsilon$ for all $j$. If $\Pi$ is a subset of $\widehat{A}, A$ is $\Pi$-inner quasidiagonal if $A$ is $z$-inner quasidiagonal, where $z$ is the support projection of $\Pi$ in the center of $A^{* *}$.

$A$ is inner quasidiagonal if and only if $A$ is $1_{A^{* *}-\text { inner quasidiagonal. } A \text { is }}$ $z$-inner quasidiagonal if and only if $A$ is $\Pi_{z}$-inner quasidiagonal, where $\Pi_{z}$ is the set of irreducible representations of $A$ with central support $\leq z$.

Example 3.14. Let $A$ be a $C^{*}$-algebra with a quasidiagonal faithful irreducible representation $\pi$, and let $z$ be the support projection of $\pi$ in $A^{* *}$. Then $A$ is $z$-inner quasidiagonal, and $z A^{* *}$ is a type I factor. More generally, if $\left\{\pi_{j}\right\}$ is a separating family of quasidiagonal irreducible representations of $A$, and $z$ is the support projection of $\oplus \pi_{j}$, then $A$ is $z$-inner quasidiagonal.

There are versions of 3.6-3.8 for $z$-inner or П-inner quasidiagonality, although 3.7(iv) must be weakened (but see 3.18). If $A$ is a $C^{*}$-algebra, $B$ a $C^{*}$-algebra, and $\Pi$ a subset of $\widehat{A}$, let $\left.\Pi\right|_{B}$ be the subset of $\widehat{B}$ consisting of all irreducible representations (actually, not just weakly) contained in $\left.\pi\right|_{B}$ for some $\pi \in \Pi$.

Proposition 3.15. Let $A$ be a $C^{*}$-algebra and $\Pi$ a subset of $\widehat{A}$. If $A$ is $\Pi$-inner quasidiagonal and $B$ is separable $C^{*}$-subalgebra of $A$, then there is a separable $C^{*}$-subalgebra $E$ of $A$, containing $B$, which is $\left.\Pi\right|_{E}$-inner quasidiagonal.

Proposition 3.16. Let $A$ be a separable $C^{*}$-algebra, and $\Pi$ a subset of $\widehat{A}$. The following are equivalent:

(i) $A$ is П-inner quasidiagonal.

(ii) Given $x_{1}, \ldots, x_{m} \in A$ and $\varepsilon>0$, there is an irreducible representation $\pi \in \Pi$ on a Hilbert space $\mathbf{H}$ and a finite-rank projection $p \in \mathbf{B}(\mathbf{H})$ such that $\left\|\left[p, \pi\left(x_{j}\right)\right]\right\|<\varepsilon$ for $1 \leq j \leq m$ and $\left\|\left[p \pi\left(x_{j}\right) p\right]\right\|>\left\|x_{1}\right\|-\varepsilon$.

(iii) There is a sequence of irreducible representations $\left\langle\pi_{n}\right\rangle$ in $\Pi$ on Hilbert spaces $\mathbf{H}_{n}$, and finite-rank projections $p_{n} \in \mathbf{B}\left(\mathbf{H}_{n}\right)$, such that $\left\|\left[p_{n}, \pi_{n}(x)\right]\right\| \rightarrow 0$ and $\lim \sup \left\|p_{n} \pi_{n}(x) p_{n}\right\|=\|x\|$ for all $x \in A$.

(iv) There is a sequence of irreducible representations $\left\langle\pi_{n}\right\rangle$ in $\Pi$ on Hilbert spaces $\mathbf{H}_{n}$, and finite-rank projections $p_{n} \in \mathbf{B}\left(\mathbf{H}_{n}\right)$, such that $\left\|\left[p_{n}, \pi_{n}(x)\right]\right\| \rightarrow 0$ and $\lim \sup \left\|p_{n} \pi_{n}(x) p_{n}\right\|=\|x\|$ for all $x \in A$, such that any GCR representation occuring more than once (up to equivalence) in the sequence is quasidiagonal. 
Proposition 3.17. Let $A$ be a $C^{*}$-algebra, $z$ a central projection in $A^{* *}$. Then, for any $k, A$ is z-inner quasidiagonal if and only if $M_{k}(A)=A \otimes \mathbf{M}_{k}$ is $(z \otimes 1)$-inner quasidiagonal. The projections for $A \otimes \mathbf{M}_{k}$ may be chosen of the form $p \otimes 1_{k}$, where $p$ is in the socle of $z A^{* *}$.

Actually, $z$-inner or $\Pi$-inner quasidiagonality is not really a stronger condition than inner quasidiagonality for separable $C^{*}$-algebras, as the next result shows. This is a generalization of 2.5 , and is closely related to 3.7 .

Theorem 3.18. Let $A$ be a separable inner quasidiagonal $C^{*}$-algebra, and $\Pi$ a separating set of mutually inequivalent irreducible representations of $A$. Then $A$ is П-inner quasidiagonal.

Lemma 3.19. Let $A$ be a $C^{*}$-algebra, $\Pi$ a faithful family of irreducible representations of $A, \pi_{1}, \ldots, \pi_{n} \in \Pi, p_{k}$ a finite-rank projection in $\pi_{k}(A)^{\prime \prime}$ for $1 \leq k \leq n, X$ a finite subset of $A$, and $\eta>0$. If $\rho$ is an representation of $A$ not equivalent to any $\pi \in \Pi$, and $q$ a finite-rank projection on $\mathbf{H}_{\rho}$, then there is a $\pi \in \Pi$ and finite-rank projection $p$ on $\mathbf{H}_{\pi}$, with $p \perp p_{k}$ if $\pi \sim \pi_{k}$, and an isometry $z$ from $q \mathbf{H}_{\rho}$ onto $p \mathbf{H}_{\pi}$, such that $\left\|q \rho(x) q-z^{*} p \pi(x) p z\right\|<\eta$ for all $x \in X$.

Proof. First suppose $\pi_{0} \in \Pi$ is GCR, with kernel $J$, and let $K=\pi_{0}^{-1}(\mathbf{K})$. If $I=\cap\left\{\operatorname{ker} \pi: \pi \in \Pi, \pi \neq \pi_{0}\right\}$, we have $I \cap J=0$, so if $L \in \operatorname{Prim}(A), L \neq J$, then either $I \subseteq L$ or $K \subseteq L$. Thus if $p$ is any finite-rank projection on $\mathbf{H}_{\pi_{0}}$, and $\rho$ is an irreducible representation of $A$ not equivalent to $\pi_{0}$, then for any $x \in A$ we have

$$
\|\rho(x)\| \leq \max \left\{\left\|(1-p) \pi_{0}(x)(1-p)\right\|, \max \left\{\|\pi(x)\|: \pi \in \Pi, \pi \not \pi_{0}\right\}\right\} .
$$

This formula also holds if $\pi_{0}$ is not GCR, for any $\rho$ and $p$, since then the right-hand side is equal to $\|x\|$.

Now let $\rho$ and $q$ be as in the statement of the lemma, with $r=\operatorname{rank} q$. By replacing $A$ by $M_{r}(A)$ and using the standard identifications of [ $\left.\mathbf{P a}, \S 5\right]$ (cf. $[\mathbf{B K b}, 4.3]$ ), we may assume $r=1$. If $p_{1}, \ldots, p_{n}$ are given, let $S$ be the set of vector states coming from representations in $\Pi$, where only vector states from $\pi_{k}$ coming from vectors orthogonal to $p_{k}$ are included. Then by the first part of the proof, for any $x=x^{*}$ in $A$ we have $\|\rho(x)\| \leq \sup _{f \in S}|f(x)|$. So by the bipolar theorem, the weak-* closure of $S$ contains all pure states of $\rho(A)$, proving the lemma.

Proof of Theorem 3.18. Let $x_{1}, \ldots, x_{m} \in A$, of norm 1 , and $0<\varepsilon<1$. Choose mutually inequivalent irreducible representations $\rho_{1}, \ldots, \rho_{n}$ of $A$ and finite-rank projections $q_{1}, \ldots, q_{n}$ such that $\left\|\left[q_{k}, \rho_{k}\left(x_{j}\right)\right]\right\|<\varepsilon / 2 m$ for all $j$ and $k$ and such that, for each $j$, there is at least one $k$ with $\left\|q_{k} \rho_{k}\left(x_{j}\right) q_{k}\right\|>$ $\left\|x_{j}\right\|-\varepsilon / 2(2.3)$. Define new pairs $\left(\pi_{k}, p_{k}\right)$ for $1 \leq k \leq n$ inductively as follows, with each $\pi_{k}$ in $\Pi$. Suppose $\left(\pi_{1}, p_{1}\right), \ldots,\left(\pi_{k-1}, p_{k-1}\right)$ have been defined. If $\rho_{k}$ is in $\Pi$, set $\left(\pi_{k}, p_{k}\right)=\left(\rho_{k}, q_{k}\right)$. Otherwise, choose $\pi, p, z$ as 
in 3.19 for $\eta=(\varepsilon / 2 m)^{2}, X=\left\{x_{j}, x_{j}^{*} x_{j}, x_{j} x_{l}^{*}: 1 \leq j \leq m\right\}$, and $\rho=\rho_{k}$, $q=q_{k}$, such that $p \perp p_{i}$ for all $i<k$ for which $\pi=\pi_{i}$. Then $\left\|p \pi\left(x_{j}\right) p\right\|>$ $\left\|q_{k} \rho_{k}\left(x_{j}\right) q_{k}\right\|-\varepsilon / 2 m$ for all $j$. Also, for each $j$,

$$
\begin{aligned}
\| p & \pi\left(x_{j}\right)^{*} \pi\left(x_{j}\right) p-p \pi\left(x_{j}\right)^{*} p \pi\left(x_{j}\right) p \| \\
\leq & \left\|z^{*} p \pi\left(x_{j}^{*} x_{j}\right) p z-q \rho\left(x_{j}^{*} x_{j}\right) q\right\| \\
& +\left\|q \rho\left(x_{j}^{*} x_{j}\right) q-q \rho\left(x_{j}\right)^{*} q \rho\left(x_{j}\right) q\right\| \\
& +\left\|q \rho\left(x_{j}\right)^{*} q \rho\left(x_{j}\right) q-z^{*} p \pi\left(x_{j}\right)^{*} p z q \rho\left(x_{j}\right) q\right\| \\
& +\left\|z^{*} p \pi\left(x_{j}\right)^{*} p z q \rho\left(x_{j}\right) q-z^{*} p \pi\left(x_{j}\right)^{*} p \pi\left(x_{j}\right) p z\right\| \\
< & \left(\frac{\varepsilon}{2 m}\right)^{2}+\left(\frac{\varepsilon}{2 m}\right)^{2}+\left(\frac{\varepsilon}{2 m}\right)^{2}+\left(\frac{\varepsilon}{2 m}\right)^{2}=\left(\frac{\varepsilon}{m}\right)^{2}
\end{aligned}
$$

(see 3.3 for the second term). Similarly, $\left\|p \pi\left(x_{j} x_{j}^{*}\right) p-p \pi\left(x_{j}\right) p \pi\left(x_{j}\right)^{*} p\right\|<$ $(\varepsilon / m)^{2}$, so $\left\|\left[p, \pi\left(x_{j}\right)\right]\right\|<\varepsilon / m$ by 3.3 . Set $\left(\pi_{k}, p_{k}\right)=(\pi, p)$.

We have now obtained a set $\left\{\left(\pi_{k}, p_{k}\right): 1 \leq k \leq n\right\}$ of representations in $\Pi$ and finite-rank projections such that $\left\|\left[p_{k}, \pi_{k}\left(x_{j}\right)\right]\right\|<\varepsilon / m$ for all $j$ and $k$ and such that, for each $j$, there is at least one $k$ for which $\left\|\left[p_{k}, \pi_{k}\left(x_{j}\right) p_{k}\right]\right\|>$ $\left\|x_{j}\right\|-\varepsilon$. The $\pi_{k}$ are, however, not necessarily distinct. Suppose, for some set $F$, each $\pi_{k}$ for $k \in F$ is equal to a representation $\pi_{0}$ in $\Pi$. Then $\left\{p_{k}: k \in F\right\}$ are mutually orthogonal, and if $p_{0}=\sum_{k \in F} p_{k}$, then

$$
\begin{aligned}
\left\|\left[p_{0}, \pi_{0}\left(x_{j}\right)\right]\right\| & \leq \sum_{k \in F}\left\|\left[p_{k}, \pi_{k}\left(x_{j}\right)\right]\right\|<\frac{\varepsilon|F|}{m} \leq \varepsilon \\
\left\|p_{0} \pi_{0}\left(x_{j}\right) p_{0}\right\| & \geq \max _{k \in F}\left\|p_{k} \pi_{k}\left(x_{j}\right) p_{k}\right\|
\end{aligned}
$$

for all $j$, so $\oplus\left(\pi_{k}, p_{k}\right)$ may be replaced by $\left(\pi_{0}, p_{0}\right) \oplus \oplus_{k \in F}\left(\pi_{k}, p_{k}\right)$. After finitely many such procedures, a direct sum of mutually inequivalent irreducible representations in $\Pi$ is obtained, satisfying the definition of $\Pi$-inner quasidiagonality for $x_{1}, \ldots, x_{m}, \varepsilon$.

\section{The Main Theorem.}

We first show that strong NF algebras are inner quasidiagonal. We begin by recalling one of the characterizations of strong NF algebras from $[\mathbf{B K b}]$; we state a slightly refined form for later use. The proof is essentially identical to the proof of $[\mathbf{B K b}, 6.1 .1]$ (note that that proof works throughout if the finite-dimensional algebras are restricted to be in a given class $\mathbf{B}$ ).

Proposition 4.1. Let $\mathbf{B}$ be a set of finite-dimensional $C^{*}$-algebras, and $A$ a separable $C^{*}$-algebra. Then the following are equivalent:

(i) $A$ can be written as $\underset{\longrightarrow}{\lim }\left(A_{n}, \phi_{m, n}\right)$ for a generalized inductive system $\left(A_{n}, \phi_{m, n}\right)$ with each $\vec{A}_{n}$ isomorphic to an algebra in $\mathbf{B}$ and each $\phi_{m, n}$ a complete order embedding (completely positive complete isometry). 
(ii) For every $x_{1}, \ldots, x_{m} \in A$ and $\varepsilon>0$, there is a $B \in \mathbf{B}$, elements $b_{1}, \ldots, b_{m} \in B$, and a complete order embedding $\phi: B \rightarrow A$ with $\left\|x_{j}-\phi\left(b_{j}\right)\right\|<\varepsilon$ for all $j$.

The strong NF algebras are exactly the $A$ for which these conditions hold for $\mathbf{B}$ the set of all finite-dimensional $C^{*}$-algebras. We may take condition (ii) (with $\mathbf{B}$ the set of all finite-dimensional $C^{*}$-algebras) to be the definition of a strong NF algebra even in the nonseparable case.

\section{Proposition 4.2. Every strong NF algebra is inner quasidiagonal.}

Proof. Let $A$ be a strong NF algebra, $x_{1}, \ldots, x_{m} \in A$, and $\varepsilon>0$. Choose a finite-dimensional $C^{*}$-algebra $B$, elements $b_{1}, \ldots, b_{m} \in B$, and a complete order embedding $\phi: B \rightarrow A$ such that if $y_{j}=\phi\left(b_{j}\right)$, then $\left\|x_{j}-y_{j}\right\|<\varepsilon / 2$ for all $j$. Let $D$ be the $C^{*}$-subalgebra of $A$ generated by $\phi(B)$. By $[$ CE1, 4.1] (cf. [BKb, 4.2.2]), there is a *-homomorphism $\pi$ from $D$ onto $B$ with $\pi\left(y_{j}\right)=b_{j}$ for all $j$. If $B=B_{1} \oplus \cdots \oplus B_{n}$ with each $B_{i}$ a full matrix algebra, and $\pi=\pi_{1} \oplus \cdots \oplus \pi_{n}$, then $\pi_{i}$ can be regarded as an irreducible representation of $D$ on a finite-dimensional Hilbert space $\mathbf{H}_{i}$. Extend $\pi_{i}$ to an irreducible representation $\widetilde{\pi}_{i}$ of $A$ on a larger Hilbert space $\widetilde{\mathbf{H}}_{i}$, and let $p_{i}$ be the projection of $\widetilde{\mathbf{H}}_{i}$ onto $\mathbf{H}_{i}$. The $\widetilde{\pi}_{i}$ are not in general inequivalent; we may assume that $\widetilde{\pi}_{1}, \ldots, \widetilde{\pi}_{r}$ are a set of representatives for the equivalence classes. Set $\widetilde{\mathbf{H}}=\widetilde{\mathbf{H}}_{1} \oplus \cdots \oplus \widetilde{\mathbf{H}}_{r}$ and $\widetilde{\pi}=\widetilde{\pi}_{1} \oplus \cdots \oplus \widetilde{\pi}_{r}$. For $i>r$, choose $k \leq r$ with $\widetilde{\pi}_{i} \approx \widetilde{\pi}_{k}$ and identify $\widetilde{\mathbf{H}}_{i}$ with $\widetilde{\mathbf{H}}_{k}$, and $p_{i}$ with the corresponding projection on $\widetilde{\mathbf{H}}_{k}$. Let $p \in \mathbf{B}(\widetilde{\mathbf{H}})$ be the sum of the $p_{i}$ (note that for a fixed $k$ the $p_{i}$ on $\widetilde{\mathbf{H}}_{k}$ are orthogonal since the $\pi_{i}$ are disjoint). Then $p \in \widetilde{\pi}(A)^{\prime \prime}$; and for each $j,\left[p, \widetilde{\pi}\left(y_{j}\right)\right]=0$. so $\left\|\left[p, \widetilde{\pi}\left(x_{j}\right)\right]\right\| \leq 2\left\|x_{j}-y_{j}\right\|<\varepsilon$. For each $j$ we have $\left\|p \tilde{\pi}\left(y_{j}\right) p\right\|=\left\|y_{j}\right\|$ (since $\left.\left\|\pi\left(y_{j}\right)\right\|=\left\|y_{j}\right\|\right)$; so

$$
\left\|p \widetilde{\pi}\left(x_{j}\right) p\right\| \geq\left\|y_{j}\right\|-\left\|x_{j}-y_{j}\right\|>\left\|y_{j}\right\|-\varepsilon / 2>\left\|x_{j}\right\|-\varepsilon .
$$

The next proposition gives an important technical characterization of inner quasidiagonality.

Proposition 4.3. Let $A$ be a $C^{*}$-algebra, and $z$ a central projection in $A^{* *}$. Then $A$ is z-inner quasidiagonal if and only if, for any $x_{1}, \ldots, x_{m} \in A$, completely positive contraction $\phi: A \rightarrow \mathbf{M}_{n}$, and $\varepsilon>0$, there is a projection $p$ in the socle of $z A^{* *}$ with $\left\|\left[p, x_{j}\right]\right\|<\varepsilon$ for all $j$, and a completely positive contraction $\psi: p A^{* *} p \rightarrow \mathbf{M}_{n}$ with $\left\|\phi\left(x_{j}\right)-\psi\left(p x_{j} p\right)\right\|<\varepsilon$ for all $j$.

Proof. The "if" part is obvious (consider the case $n=1$ ). Conversely, suppose $A$ is $z$-inner quasidiagonal; we may assume $A$ is unital. Fix $x_{1}, \ldots, x_{m} \in$ $A$ and $\varepsilon>0$. By 3.15 we may assume $A$ is separable. Then we may assume there is a set $\Pi$ of irreducible representations as in 3.16(iv) such that $z$ is the support projection of $\Pi$. Because of 3.17 and the identifications described 
in $[\mathbf{P a}, \S 5]$ ( $\mathrm{cf}$. [ $\mathbf{B K b}, 4.3])$, we may assume $n=1$. For each $\delta>0$ let $S_{\delta}$ be the weak-* closure of the set of all states $\omega$ of $A$ of the form $\omega(x)=\psi(p x p)$, where $p$ is in the socle of $z A^{* *},\left\|\left[p, x_{j}\right]\right\|<\delta$ for all $j$, and $\psi$ is a state on $p A^{* *} p$. We want to show that $S_{\varepsilon}$ is the entire state space of $A$. For any $\delta, S_{\delta}$ is norming for $A$, i.e., for $x=x^{*} \in A, \sup \left\{|\phi(x)|: \phi \in S_{\delta}\right\}=\|x\|$ by $\Pi$-inner quasidiagonality. Therefore, if $\|x\| \leq 1$ and $\phi(x) \geq 0$ for all $\phi \in S_{\delta}$, then $\phi(1-x) \leq 1$ for all $\phi \in S_{\delta}$, so $\|1-x\| \leq 1, x \geq 0$. Thus $S_{\delta}$ contains all pure states of $A$ by [Dx, 3.4.1], so it suffices to show that a convex combination of two elements of $S_{\delta / 2}$ is in $S_{\delta}$. So let $p_{1}, p_{2}$ be projections in the socle of $z A^{* *},\left\|\left[p_{i}, x_{j}\right]\right\|<\delta / 2$ for $i=1,2,1 \leq j \leq n$, and $\omega_{i}$ states on $A$ of the form $\omega_{i}(x)=\psi_{i}\left(p_{i} x p_{i}\right)$ for states $\psi_{i}$ on $p_{i} A^{* *} p_{i}$. As in 2.3, there are representations $\pi_{i}$ of $A$, each of which is a direct sum of mutually inequivalent irreducible representations in $\Pi$, such that $\pi_{i}\left(p_{i}\right) \pi_{i}(A) \pi_{i}\left(p_{i}\right)=p_{i} A p_{i}$, and $\omega_{i}$ is a linear combination of vector states from vectors in the range of $\pi\left(p_{i}\right)$. If $0<\lambda<1$ is fixed, we must show that $\omega=\lambda \omega_{1}+(1-\lambda) \omega_{2}$ is approximately of the same form.

The difficulty comes when one or more of the irreducible subrepresentations of $\pi_{1}$ is equivalent to a subrepresentation of $\pi_{2}$. By the choice of $\Pi$, any such representation $\rho$ is either quasidiagonal or not GCR. We will separately work within each such $\rho$, so fix $\rho$, on a Hilbert space $\mathbf{H}$.

If $\rho$ is quasidiagonal, identify the components of $\pi\left(p_{i}\right)(i=1,2)$ in $\rho$ with $q_{i} \in \mathbf{B}(\mathbf{H})$. Then, for any $\eta>0$, there is a finite-rank projection $r$ such that $\left\|q_{i}-r q_{i}\right\|<\eta(i=1,2)$ and $\left\|\left[r, \rho\left(x_{j}\right)\right]\right\|<\varepsilon$ for $1 \leq j \leq m$; the component of $\omega$ corresponding to $\rho$ can thus be approximated within $\eta$ in norm by a convex combination of vector states in the range of $r$, and such states are in $S_{\varepsilon}$.

Now suppose $\rho$ is not GCR, i.e., $\rho(A) \cap \mathbf{K}=\{0\}$. Identify the subrepresentation $\rho_{1}$ of $\pi_{1}$ equivalent to $\rho$ with $\rho$, giving a projection $q_{1}$; then the component of $\omega_{1}$ corresponding to $\rho$ is a convex combination of vector states in the range of $q_{1}$. Let $\rho_{2}$ on $\mathbf{H}_{2}$ be the subrepresentation of $\pi_{2}$ equivalent to $\rho$, and $r_{2}$ the corresponding projection. By [Vo1, Lemma 1], for any $y_{1}, \ldots, y_{r} \in A$ and $\eta>0$, there is a unitary $u$ from $\mathbf{H}_{2}$ to $\mathbf{H}$ and a finite-rank projection $q_{2} \in \mathbf{B}(\mathbf{H})$ orthogonal to $q_{1}$ with $q_{2}=u r_{2} u^{*}$ and $\left\|q_{2} \rho\left(x_{j}\right) q_{2}-u r_{2} \rho_{2}\left(x_{j}\right) r_{2} u^{*}\right\|<\eta,\left\|q_{2} \rho\left(y_{k}\right) q_{2}-u r_{2} \rho_{2}\left(y_{k}\right) r_{2} u^{*}\right\|<\eta$ for all $j, k$. Thus every weak-* neighborhood of the component of $\omega_{2}$ in $\rho_{2}$ contains a state $\widetilde{\omega}_{2}$ which is a convex combination of vector states in the range of some such $q_{2}$, and $\widetilde{\omega}_{2}$ is in $S_{\delta / 2}$ for sufficiently small $\eta$. Then the component of $\omega$ corresponding to $\rho$ is approximated by a convex combination of vector states in the range of $q_{1}+q_{2}$, and $\left\|\left[q_{1}+q_{2}, x_{j}\right]\right\| \leq\left\|\left[q_{1}, x_{j}\right]\right\|+\left\|\left[q_{2}, x_{j}\right]\right\|<\delta$ for all $j$, so $\omega \in S_{\delta}$.

Theorem 4.4. Let $A$ be a nuclear $C^{*}$-algebra, and $z$ a central projection in $A^{* *}$. If $A$ is z-inner quasidiagonal, then $A$ satisfies condition (ii) of 4.1 with 
$\mathbf{B}=\left\{p A p: p\right.$ is in the socle of $\left.z A^{* *}\right\}$. So if $A$ is separable, then $A$ can be written as $\lim _{\longrightarrow}\left(A_{n}, \phi_{m, n}\right)$ for a generalized inductive system $\left(A_{n}, \phi_{m, n}\right)$ where each $A_{n}$ is isomorphic to $p_{n} A^{* *} p_{n}$ for some $p_{n}$ in the socle of $z A^{* *}$, and each $\phi_{m, n}$ is a complete order embedding.

Putting together 4.4 with 4.2 we obtain:

Theorem 4.5. Let $A$ be a separable $C^{*}$-algebra. Then $A$ is a strong $\mathrm{NF}$ algebra if and only if $A$ is nuclear and inner quasidiagonal.

Proof of Theorem 4.4. Suppose $A$ is nuclear and $z$-inner quasidiagonal. Let $x_{1}, \ldots, x_{m} \in A$ and $\varepsilon>0$. Choose a matrix algebra $\mathbf{M}_{n}$ and completely positive contractive maps $\alpha: A \rightarrow \mathbf{M}_{n}$ and $\beta: \mathbf{M}_{n} \rightarrow A$ such that $\left\|\beta \circ \alpha\left(x_{j}\right)-x_{j}\right\|<\varepsilon / 7$ for $1 \leq j \leq m$. Then by 4.3 choose $p$ in the socle of $z A^{* *}$ with $\left\|\left[p, x_{j}\right]\right\|<\varepsilon / 7$ and $\sigma: p A p \rightarrow \mathbf{M}_{n}$ a completely positive contraction such that $\left\|\sigma\left(p x_{j} p\right)-\alpha\left(x_{j}\right)\right\|<\varepsilon / 7$ for $1 \leq j \leq m$. Set $B=p A p$ and $\omega=\beta \circ \sigma: B \rightarrow A$. Then $\left\|\omega\left(p x_{j} p\right)-x_{j}\right\|<\varepsilon / 7$ for all $j$.

We have $d\left(x_{j}, N_{p}\right)=\left\|\left[p, x_{j}\right]\right\|$ for each $j$ by 3.5 ; let $y_{j} \in N_{p}$ with $\left\|x_{j}-y_{j}\right\|<\varepsilon / 7$. Then $\left\|\omega\left(p y_{j}\right)-y_{j}\right\|<4 \varepsilon / 7$ for all $j$. The map $x \rightarrow p x=p x p$ is a ${ }^{*}$-homomorphism from $N_{p}$ onto $B$; let $J$ be the kernel, $\psi: B \rightarrow N_{p}$ a completely positive contractive cross section for the quotient map, and $\left\{e_{i}\right\}$ a quasicentral approximate identity for $J$ in $N_{p}$. For each $i$, define $\phi_{i}: B \rightarrow A$ by

$$
\phi_{i}(b)=\left(1-e_{i}\right)^{1 / 2} \psi(b)\left(1-e_{i}\right)^{1 / 2}+e_{i}^{1 / 2} \omega(b) e_{i}^{1 / 2} .
$$

For each $i, \phi_{i}$ is a complete order embedding since $p \phi_{i}(b) p=b$ for all $b \in B$. For $i$ sufficiently large, $\left\|y_{j}-\left(\left(1-e_{i}\right)^{1 / 2} y_{j}\left(1-e_{i}\right)^{1 / 2}+e_{i}^{1 / 2} y_{j} e_{i}^{1 / 2}\right)\right\|<\varepsilon / 7$ for all $j$ since $\left\{e_{i}\right\}$ is quasicentral. We also have

$$
\left\|\left(1-e_{i}\right)^{1 / 2}\left(y_{j}-\psi\left(p y_{j}\right)\right)\left(1-e_{i}\right)^{1 / 2}\right\|<\varepsilon / 7
$$

for each $j$, for $i$ large, since $y_{j}-\psi\left(p y_{j}\right) \in J$. Thus, for $i$ sufficiently large, we have, for all $1 \leq j \leq m$,

$$
\begin{aligned}
& \left\|x_{j}-\phi_{i}\left(p y_{j}\right)\right\| \\
& \leq \quad\left\|x_{j}-y_{j}\right\|+\left\|y_{j}-\left(\left(1-e_{i}\right)^{1 / 2} y_{j}\left(1-e_{i}\right)^{1 / 2}+e_{i}^{1 / 2} y_{j} e_{i}^{1 / 2}\right)\right\| \\
& \quad+\left\|\left(1-e_{i}\right)^{1 / 2}\left(y_{j}-\psi\left(p y_{j}\right)\right)\left(1-e_{i}\right)^{1 / 2}\right\|+\left\|e_{i}^{1 / 2}\left(y_{j}-\omega\left(p y_{j}\right)\right) e_{i}^{1 / 2}\right\| \\
& \quad<\frac{\varepsilon}{7}+\frac{\varepsilon}{7}+\frac{\varepsilon}{7}+\frac{4 \varepsilon}{7}=\varepsilon,
\end{aligned}
$$

so $A$ satisfies condition (ii) of 4.1. If $A$ is separable, the last statement of 4.4 follows from 4.1 .

The following diagram summarizes the maps used in the proof. 


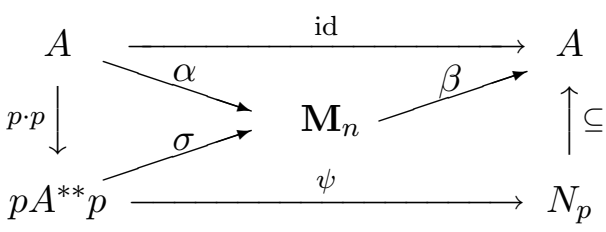

5. Corollaries.

Corollary 5.1. A separable nuclear $C^{*}$-algebra with a separating family of quasidiagonal irreducible representations is a strong NF algebra. In particular, every separable nuclear residually finite-dimensional $C^{*}$-algebra is a strong NF algebra, and every separable nuclear strongly quasidiagonal $C^{*}$ algebra is a strong NF algebra.

Corollary 5.2. Let $A$ be a separable subhomogeneous $C^{*}$-algebra, and suppose each irreducible representation of $A$ is of dimension $\leq k$. Then $A$ is a strong NF algebra, and has a strong NF system $\left(A_{n}, \phi_{m, n}\right)$ where each $A_{n}$ is a (finite) direct sum of matrix algebras of size not more than $k \times k$.

Since the class of strong NF algebras is closed under inductive limits [BKb, 6.1.3], we obtain:

Corollary 5.3. Every (separable) approximately subhomogeneous $C^{*}$-algebra is a strong NF algebra.

Corollary 5.4. If $A$ is separable, nuclear, and prime, then $A$ is a strong NF algebra if and only if some (hence every) faithful irreducible representation of $A$ is quasidiagonal.

Corollary 5.5. Every antiliminal prime NF algebra is a strong NF algebra. Every simple NF algebra is a strong NF algebra.

Example 5.6. The examples of 2.7 are NF but not strong NF.

Corollary 5.7. Let $A$ be a separable nuclear $C^{*}$-algebra. The following are equivalent:

(i) Every quotient of $A$ is a strong NF algebra.

(ii) Every primitive quotient of $A$ is a strong NF algebra.

(iii) Every irreducible representation of $A$ is quasidiagonal.

(iv) A is strongly quasidiagonal.

Corollary 5.8. Let $A$ be any $N F$ algebra, and let $B$ be a split essential extension of $A$ by $\mathbf{K}$. Then $B$ is a strong NF algebra. So $A$ can be embedded as a $C^{*}$-subalgebra of a strong $N F$ algebra $B$ with a retraction (homomorphic conditional expectation) from $B$ onto $A$. In particular, $A$ is a quotient of a strong NF algebra. So every separable nuclear $C^{*}$-algebra is a quotient of a strong NF algebra $[\mathbf{B K b}, 6.1 .8]$. 
We can obtain a refinement of 5.4-5.5.

Definition 5.9. A strong NF algebra is of monomial type if it can be written as $\lim _{\longrightarrow}\left(A_{n}, \phi_{m, n}\right)$, with each $A_{n}$ a single matrix algebra and each $\phi_{m, n}$ a complete order embedding.

We have used the terminology "monomial type" instead of "UHF type" or "matroid type" since the class of AF algebras which are strong NF of monomial type is considerably larger than the class of UHF or matroid $C^{*}$ algebras. (In fact, an AF algebra is strong NF of monomial type if and only if it is prime.)

Proposition 5.10 (cf. [Dx, 1.9.13]). $A C^{*}$-algebra $B$ is prime if and only if, for every nonzero $x, y \in B$, there is an irreducible representation $\pi$ of $B$ with $\pi(x)$ and $\pi(y)$ both nonzero.

Proof. If $I$ and $J$ are nonzero ideals of $B$ with $I \cap J=0$, then every irreducible representation of $A$ must annihilate either $I$ or $J$, so if $x \in I$ and $y \in J$ are nonzero, then no irreducible representation of $B$ can be nonzero on both $x$ and $y$. Conversely, if $B$ is prime, and $x, y$ are nonzero elements of $B$, then there is a $z \in B$ with $x z y \neq 0$ (otherwise the two-sided ideals generated by $x$ and $y$ annihilate each other); if $\pi$ is an irreducible representation of $B$ with $\pi(x z y) \neq 0$, then $\pi(x)$ and $\pi(y)$ are both nonzero.

Remark 5.11. The second half of the proof can be simplified if $B$ is primitive (e.g., if $B$ is separable). It is still an open question whether every prime $C^{*}$-algebra is primitive.

Proposition 5.12. A strong NF algebra of monomial type is prime.

Proof. If $A$ is strong NF of monomial type and $x_{1}, x_{2}$ are nonzero elements of $A$, then by 4.1 there is a complete order embedding $\phi$ of a full matrix algebra $B$ into $A$ such that $\left\|x_{j}-\phi\left(b_{j}\right)\right\|<\left\|x_{j}\right\| / 2$ for $j=1,2$, for some $b_{j} \in B . \phi^{-1}$ extends to an irreducible representation $\tilde{\pi}$ of $A$ as in proof of 4.2 , and $\widetilde{\pi}\left(x_{j}\right) \neq 0$ for $j=1,2$.

Theorem 5.13. Let $A$ be a prime separable nuclear $C^{*}$-algebra. Then the following are equivalent:

(i) $A$ is a strong NF algebra.

(ii) A is a strong NF algebra of monomial type.

(iii) For some faithful irreducible representation $\pi, \pi(A)$ is a quasidiagonal $C^{*}$-algebra of operators.

(iv) For every faithful irreducible representation $\pi, \pi(A)$ is a quasidiagonal $C^{*}$-algebra of operators.

In particular, every antiliminal prime NF algebra and every simple NF algebra is a strong NF algebra of monomial type. 
Proof. (ii) $\Rightarrow$ (i) and (iv) $\Rightarrow$ (iii) are trivial; (i) $\Rightarrow$ (iv) by 2.5 and 4.2 , and (iii) $\Rightarrow$ (ii) by 4.4 , letting $z$ be the support of $\pi$ in $A^{* *}$ (cf. 3.14).

We have the following versions of 2.9-2.10:

Proposition 5.14. Let $A$ be a (separable) $C^{*}$-algebra, and $J_{1}, J_{2}$ ideals of $A$. Set $J=J_{1} \cap J_{2}$. If $A / J_{1}$ and $A / J_{2}$ are strong $N F$ algebras, then $A / J$ is strong NF.

Proof. In light of 2.9 it suffices to note that if $A / J_{1}$ and $A / J_{2}$ are nuclear, then $A / J$ is nuclear. This can be seen in several ways. Perhaps the easiest is to use the fact that a separable $C^{*}$-algebra $B$ is nuclear if and only if every factor representation of $B$ generates an injective factor, and note that every factor representation of $A / J$ factors though either $A / J_{1}$ or $A / J_{2}$. Alternatively, $A / J$ is an extension of $A / J_{1}$ by $J_{1} / J$, and $J_{1} / J \cong\left(J_{1}+J_{2}\right) / J_{2}$, which is nuclear since it is an ideal in $A / J_{2}$.

Corollary 5.15. A separable nuclear $C^{*}$-algebra $A$ is a strong $N F$ algebra if (and only if) $A$ contains a sequence $\left\langle J_{n}\right\rangle$ of ideals with $A / J_{n}$ strong $N F$ for all $n$ and $\cap J_{n}=0$.

Note that neither of the assumptions that $A$ be separable and nuclear follow from the other hypotheses of 5.15 (e.g., $A=\prod_{n} \mathbf{M}_{n}, J_{n}$ the sequences vanishing in the $n$ 'th coordinate).

The situation with an increasing sequence of ideals, and hence with inductive limits with noninjective connecting maps, is quite different. Recall that an (ordinary) inductive limit, with injective connecting maps, of strong $\mathrm{NF}$ algebras is strong $\mathrm{NF}([\mathbf{B K b}, 6.1 .3]$; this is an immediate corollary of 4.1 , or of 2.1 and 4.5$)$.

Proposition 5.16. An (ordinary) inductive limit of an inductive system of strong NF algebras with noninjective connecting maps is not necessarily strong NF.

Proof. Example 2.12 is a counterexample.

For completeness, we note the following fact, which should have been included in [BKb]:

Proposition 5.17. The class of strong NF algebras is closed under tensor products.

Proof. By 4.1 it suffices to show that, if $A_{1}, A_{2}, B_{1}, B_{2}$ are $C^{*}$-algebras, with $B_{1}, B_{2}$ finite-dimensional, and $\phi_{i}: B_{i} \rightarrow A_{i}$ are complete order embeddings, then the finite-dimensional subspace $\phi_{1}\left(B_{1}\right) \otimes \phi_{2}\left(B_{2}\right)$ of $A_{1} \otimes A_{2}$ is completely order isomorphic to a $C^{*}$-algebra. This follows immediately from [CE2, 3.1] (cf. [BKb, 4.2.1]), since if $\omega_{i}$ is an idempotent completely positive contraction from $A_{i}$ onto $\phi_{i}\left(B_{i}\right)$, then $\omega_{1} \otimes \omega_{2}$ is an idempotent completely positive contraction from $A_{1} \otimes A_{2}$ onto $\phi_{1}\left(B_{1}\right) \otimes \phi_{2}\left(B_{2}\right)$. 
Finally, the next proposition is an immediate consequence of 3.11 and [BKb, 5.3.3].

Proposition 5.18. Let $A$ be a separable $C^{*}$-algebra. Then $A$ is a strong $N F$ algebra if and only if $S A$ is strong NF, and similarly for $C A$. Thus, if $A$ is a separable nuclear $C^{*}$-algebra which is not strong $N F$ (e.g., if $A$ is not $N F)$, then $S A$ and $C A$ are $N F$ but not strong $N F$.

Using 5.18, we get examples of NF algebras which are not strong NF which are very different from those of 5.6:

Example 5.19. $\mathrm{SO}_{2}$ is an antiliminal NF algebra which is not strong NF.

\section{Appendix A.}

This appendix contains a "folklore" result that we have been unable to find in the literature. The arguments are slight variations of those of Glimm $[\mathbf{G l}]$, as presented in $[\mathbf{D x}]$. The word "ideal" will mean "closed two-sided ideal".

If $J$ is a primitive ideal in a $C^{*}$-algebra $A$, we will call $J$ a $G C R$ ideal if $J$ is the kernel of a GCR irreducible representation (one whose image contains the compact operators). The next proposition is well known and easy to prove (cf. [Dx, 4.1.10]).

Proposition A.1. Let $J$ be a primitive ideal in a separable $C^{*}$-algebra $A$. Then the following are equivalent:

(i) $J$ is a GCR ideal.

(ii) There is an ideal $K$ of $A$, containing $J$, such that $K / J$ is an essential ideal of $A / J$ isomorphic to $\mathbf{K}$.

(iii) $A / J$ is not antiliminal.

Theorem A.2. Let $J$ be a primitive ideal in a separable $C^{*}$-algebra $A$. Then the following are equivalent:

(i) $J$ is not GCR ideal.

(ii) $A / J$ is antiliminal.

(iii) $J$ is the kernel of a non-type I factor representation of $A$.

(iv) There are two inequivalent irreducible representations of $A$ with kernel $J$.

(v) There are uncountably many mutually inequivalent irreducible representations of $A$ with kernel $J$.

Proof. (i) $\Leftrightarrow$ (ii) is A.1, (v) $\Rightarrow$ (iv) is trivial, and (iv) $\Rightarrow$ (i) follows from [Dx, 4.1.10].

By replacing $A$ by $A / J$, we may and will assume that $J=0$ in the rest of the proof, to simplify notation.

(iii) $\Rightarrow(\mathrm{v})$ by a slight modification of the argument in [Dx, 9.1]: if $\pi$ is a faithful non-type-I factor representation of $A$ on a separable Hilbert 
space and $C$ is a masa in $\pi(A)^{\prime}$, the direct integral decomposition of $\pi$ as $\int^{\oplus} \pi_{x} d \mu(x)$ with respect to $C$ has almost all $\pi_{x}$ faithful and irreducible by the argument of [Dx, 9.1]. If, for a set $E$ of nonzero measure, each $\pi_{x}$ for $x \in E$ is equivalent to a fixed representation $\pi_{0}$, then by [Dx, 8.1.7] $\int_{E}^{\oplus} \pi_{x} d \mu(x)$ is a subrepresentation of $\pi$ equivalent to a multiple of $\pi_{0}$, a contradiction. Thus, for each $x$, the set $E_{x}=\left\{y: \pi_{y} \sim \pi_{x}\right\}$ has measure 0 , so there must be uncountably many such sets.

It remains to prove (ii) $\Rightarrow$ (iii). This follows from the results of Glimm if $A$ has a minimal nonzero ideal, but not directly otherwise. However, using the next three lemmas, Glimm's argument essentially works in our case.

Lemma A.3. Let $A$ be a separable primitive $C^{*}$-algebra. Then $A$ contains a decreasing sequence $\left\langle J_{n}\right\rangle$ of nonzero (not necessarily proper) ideals, such that every nonzero ideal of $A$ contains $J_{n}$ for some $n$.

Proof. This is an immediate consequence of the fact that Prim $(A)$ is a second countable $T_{0}$ space and 0 is a dense point.

Lemma A.4 (cf. [Dx, 9.3.5]). Let $B$ be an antiliminal $C^{*}$-algebra and $I$ an essential ideal in $B$. If $d \in B_{+}$of norm 1 and $0<\tau \leq 1$, then there exist $w, w^{\prime}, d^{\prime}$ in I satisfying the conclusions of [Dx, 9.3.5].

Proof. The proof is identical to the proof of $[\mathbf{D x}, 9.3 .5]$ except that $\pi$ is chosen to be an irreducible representation of $B$ which is nonzero on $I$ (this is possible since $I$ is essential and is itself an antiliminal $C^{*}$-algebra), so $\left.\pi\right|_{I}$ is irreducible, and $c$ is chosen in $I$. Then $d_{0}$ and hence $\nu$ are in $I$, so $d^{\prime}, w$, and $w^{\prime}$ are also in $I$.

Lemma A.5 (cf. [Dx, 9.3.7]). Let $B$ be a unital antiliminal $C^{*}$-algebra, $\left\langle J_{n}\right\rangle$ a decreasing sequence of essential ideals of $B$, and let $\left(s_{0}, s_{1}, \ldots\right)$ be a sequence of self-adjoint elements of $B$. Then there exist elements $\nu\left(a_{1}, \ldots, a_{k}\right)(k=1,2, \ldots)$ of $B$ satisfying all the conditions of [Dx, 9.3.7], and in addition $\nu\left(a_{1}, \ldots, a_{k}\right) \in J_{k}$ for all $k$ and all $\left(a_{1}, \ldots, a_{k}\right)$.

Proof. The proof is identical to the proof of [Dx, 9.3.7], with A.4 used (with $\left.I=J_{n+1}\right)$ in place of $[\mathbf{D x}, 9.3 .5]$.

Proof of A.2 (ii) $\Rightarrow$ (iii). Let $\left\langle J_{n}\right\rangle$ be a sequence of ideals of $A$ as in A.3. Choose elements $\nu\left(a_{1}, \ldots, a_{k}\right)$ as in A.5 (if $A$ is nonunital, work in $\widetilde{A}$ ). Choose the states $f$ and $g$ as in [Dx, 9.4]; then the representation $\pi_{g}$ is a type II factor representation of $A$. If $I=\operatorname{ker} \pi_{g}$ is nonzero, then $\pi_{g}$ is zero on $J_{n}$ for some $n$; but this is impossible since $\nu\left(a_{1}, \ldots, a_{k}\right) \in J_{n}$ and $\pi_{g}\left(\nu\left(a_{1}, \ldots, a_{k}\right)\right) \neq 0$. Thus $I=0$ and $\pi_{g}$ is faithful.

The same technique can be used to give the following version of Maréchal's refinement [Ma, §2] of Glimm's result: 
Lemma A.6. Let $A$ be a separable unital primitive antiliminal $C^{*}$-algebra. Then there is a unital sub- $C^{*}$-algebra $B$ of $A$ and ideal $J$ of $B$, such that:

(a) $B / J$ is isomorphic to the $C A R$ algebra $D$ (write $\phi: B \rightarrow D$ for the quotient map).

(b) For any cyclic representation $\pi$ of $D$, there is a faithful cyclic representation $\rho$ of $A$, and a projection $F \in \rho(B)^{\prime \prime} \cap \rho(B)^{\prime}$, of central support 1 in $\rho(A)^{\prime \prime}$, such that the subrepresentation $\rho_{1}$ of $\left.\rho\right|_{B}$ defined by $F$ is equivalent to $\pi \circ \phi$ and $\rho_{1}(B)^{\prime \prime}=F \rho(A)^{\prime \prime} F$.

Corollary A.7. Let $A$ be a separable $C^{*}$-algebra and $J$ a non-GCR primitive ideal of $A$. If $M$ is any properly infinite injective von Neumann algebra (in particular, any infinite injective factor) with separable predual, then there is a representation $\pi$ of $A$ with kernel $J$, such that $\pi(A)^{\prime \prime} \cong M$.

Note added in proof. The authors have recently shown that the converse of 2.4 is true: an inner quasidiagonal $C^{*}$-algebra has a separating family of quasidiagonal irreducible representations. As a consequence, if $A$ and $B$ are inner quasidiagonal and one of them is nuclear, then $A \otimes B$ is inner quasidiagonal (see the comment after 3.9). Some of the other arguments in this paper can be simplified using this result.

\section{References}

[BKb] B. Blackadar and E. Kirchberg, Generalized inductive limits of finite-dimensional $C^{*}$-algebra, Math. Ann., 307 (1997), 343-380.

[BoD] F. Bonsall and J. Duncan, Complete Normed Algebras, Springer-Verlag, BerlinHeidelberg-New York-Tokyo, 1973.

[Bn] L. Brown, The universal coefficient theorem for Ext and quasidiagonality, in 'Operator Algebras and Group Representations', Pitman, Boston-London-Melbourne, 1983.

[BnD] L. Brown and M. Dadarlat, Extensions of $C^{*}$-algebras and quasidiagonality, J. London Math. Soc. (2), 53 (1996), 582-600.

[CE1] M.-D. Choi and E. Effros, The completely positive lifting problem for $C^{*}$-algebras, Ann. of Math., 104 (1976), 585-609.

[CE2] _ Injectivity and operator spaces, J. Funct. Anal., 24 (1977), 156-209.

[Dx] J. Dixmier, $C^{*}$-Algebras, Gauthier-Villars, Paris, 1964; North-Holland, Amsterdam, 1977.

[Gl] J. Glimm, Type I $C^{*}$-algebras, Ann. of Math., 73 (1961), 572-612.

[Ma] O. Maréchal, Topologie et structure Borélienne sur l'ensemble des algebres de von Neumann, C.R. Acad. Sci. Paris, 276 (1973), 847-850.

[Pa] V. Paulsen, Completely Bounded Maps and Dilations, Pitman Research Notes in Math., 146 (1986).

[Pd] G. Pedersen, $C^{*}$-Algebras and their Automorphism Groups, Academic Press, London-New York-San Francisco, 1979. 
[Vo1] D. Voiculescu, A noncommutative Weyl-von Neumann theorem, Rev. Roum. Math. Pures Appl., 21 (1976), 97-113.

[Vo2] A note on quasidiagonal $C^{*}$-algebras and homotopy, Duke Math. J., 62 (1991), 267-272.

Received January 5, 1996 and revised December 20, 1998.

UNIVERSITY OF NEVADA

RENO, NV 89557

E-mail address: bruceb@math.unr.edu

INSTITUT FÜR REINE MATHEMATIK

HumboldT-UNIVERSITÄT

10099 BERLIN

GERMANY

E-mail address: kirchbrg@mathematik.hu-berlin.de 